\title{
A Copper Shield for the Reduction of X- $\gamma$ True Coincidence Summing in Gamma-ray Spectrometry
}

\author{
Jong-In Byun \\ Korea Institute of Nuclear Safety, Daejeon, Korea
}

\section{Technical Paper}

Received September 3, 2018

Revision November 1, 2018

Accepted November 30, 2018

Corresponding author: Jong-In Byun

Korea Institute of Nuclear Safety,

62 Gwahak-ro, Yuseong-gu,

Daejeon 34142, Korea

Tel: $+82-42-868-0876$

Fax: +82-42-868-0563

E-mail:k975bji@kins.re.kr

This is an Open-Access article distributed under the terms of the Creative Commons Attribution NonCommercial License (http://creativecommons.org/ licenses/by-nc/4.0) which permits unrestricted noncommercial use, distribution, and reproduction in any medium, provided the original work is properly cited.

Copyright $\odot$ 2018 The Korean Association for Radiation Protection

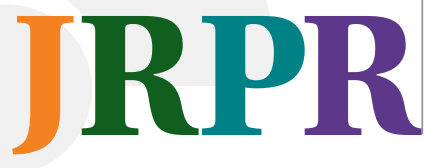

Background: Gamma-ray detectors having a thin window of a material with low atomic number can increase the true coincidence summing effects for radionuclides emitting X-rays or gamma-rays. This effect can make efficiency calibration or spectrum analysis more complicated. In this study, a $\mathrm{Cu}$ shield was tested as an X-ray filter to neglect the true coincidence summing effect by X-rays and gamma-rays in gamma-ray spectrometry, in order to simplify gamma-ray energy spectrum analysis.

Materials and Methods: A Cu shield was designed and applied to an n-type high-purity germanium detector having an X- $\gamma$ summing effect during efficiency calibration. This was tested using a commercial, certified mixed gamma-ray source. The feasibility of a $\mathrm{Cu}$ shield was evaluated by comparing efficiency calibration results with and without the shield.

Results and Discussion: In this study, the thickness of a $\mathrm{Cu}$ shield needed to avoid true coincidence summing effects due to $\mathrm{X}-\gamma$ was tested and determined to be $1 \mathrm{~mm}$, considering the detection efficiency desired for higher energy. As a result, the accuracy of the detection efficiency calibration was improved by more than $13 \%$ by reducing $\mathrm{X}-\gamma$ summing.

Conclusion: The X- $\gamma$ summing effect should be considered, along with $\gamma-\gamma$ summing, when a detection efficiency calibration is implemented and appropriate shielding material can be useful for simplifying analysis of the gamma-ray energy spectra.

Keywords: True coincidence summing, X-ray, Gamma-ray spectrometry

\section{Introduction}

A high purity germanium (HPGe) detector has been widely used for gamma-ray spectrometry because of its good resolution and there have been commercial planar, coaxial or well types. It can be fabricated to achieve higher detection efficiency especially for low energy photons. In this case, it often has a thin dead-layer, which is induced by forming an electric contact on the surface of the germanium crystal, and a thin window of a material with low atomic number such as carbon composite or beryllium. However, this can increase the summing effects for radionuclides emitting X-rays or gamma-rays in sequence within the resolving time of the preamplifier of a detector. This phenomenon is well known in gamma-ray spectrometry as a true coincidence summing effect. It can cause "summing in", in which the full-energy peak is added to, by the sum of incident photons with different energies, or "summing out", in which the full-energy peak is summed with other incident photons and recorded in a channel corresponding to a higher energy $[1,2]$. This effect can also make efficiency calibration 
or spectrum analysis more complicated, especially for true coincidence summing by X-rays and gamma-rays (hereafter referred to as ' $\mathrm{X}-\gamma$ summing').

The $\mathrm{X}-\gamma$ summing effect can be neglected by attenuating low energy X-rays in a detector's window, and this can simplify gamma-ray energy spectrum analysis because only $\gamma-\gamma$ summing is considered. Low energy $\mathrm{X}$-rays can be effectively attenuated using low atomic number materials, and $\mathrm{Cu}$ has generally been used as the most internal shielding material in gamma-ray detector shields to attenuate X-rays. Therefore, $\mathrm{Cu}$ could be useful for reduction of the $\mathrm{X}-\gamma$ summing effect in gamma-ray measurements. The purpose of this study is to test the feasibility of a $\mathrm{Cu}$ shield as an $\mathrm{X}$-ray filter to avoid the $\mathrm{X}-\gamma$ summing effect. In this paper, the characteristics of low-energy photons attenuated by $\mathrm{Cu}$, and the results of experiments and Monte Carlo simulation on the applicability of a $\mathrm{Cu}$ shield to an n-type HPGe detector, are presented.

\section{Materials and Methods}

X-rays scattered in shielding materials surrounding a detector can be summed with gamma-rays in sequence within the resolving time of the detector. Therefore, in order to reduce effectively the $\mathrm{X}-\gamma$ summing by scattered $\mathrm{X}$-rays, the $\mathrm{X}$ rays from the gamma-ray source should be absorbed predominantly by photoelectric effects in the shielding materials. Figure 1A shows the ratio of photoelectric and total mass attenuation rates for $\mathrm{Cu}$. The thickness of a $\mathrm{Cu}$ shield needs to be optimized considering the reduction rates of X-rays to

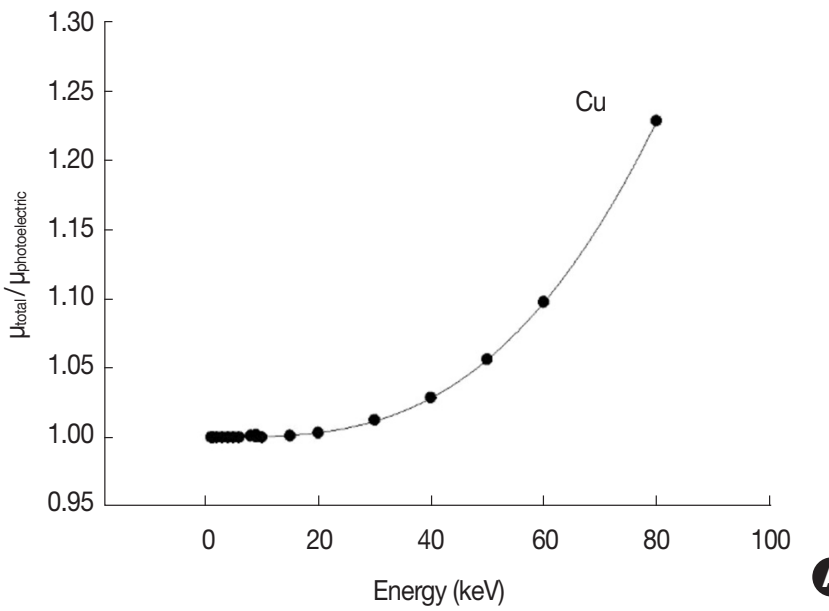

produce the $X-\gamma$ summing that minimizes the decrease of efficiency in the detection of gamma-ray energy. The linear attenuation rate of the energy lines of interest in $\mathrm{Cu}$ of varied thickness with $\mathrm{XCOM}^{1}$, was evaluated as shown in Figure 1B.

To estimate the feasibility of a $\mathrm{Cu}$ shield, one was applied to an HPGe detector to avoid the $\mathrm{X}-\gamma$ true coincidence summing effect during calibration of the absolute detection efficiency. An n-type coaxial HPGe detector with relative efficiency of $30 \%$ and commercial standard solution of mixed nuclides containing ${ }^{241} \mathrm{Am},{ }^{109} \mathrm{Cd},{ }^{57} \mathrm{Co},{ }^{139} \mathrm{Ce},{ }^{113} \mathrm{Sn},{ }^{203} \mathrm{Hg},{ }^{85} \mathrm{Sr},{ }^{137} \mathrm{Cs},{ }^{60} \mathrm{Co}$, and ${ }^{88} \mathrm{Y}$ were used in this study. The standard source was diluted with $1 \mathrm{M} \mathrm{HCl}$ and filled with a layer $10 \mathrm{~mm}$ thick in a cylindrical bottle $\left(4.8 \emptyset \times 5 \mathrm{~cm}^{3}\right)$; then measured using the HPGe detector. The radionuclides of ${ }^{139} \mathrm{Ce},{ }^{85} \mathrm{Sr}$, and ${ }^{88} \mathrm{Y}$ in the source can significantly produce $\mathrm{X}-\gamma$ summing in gammaray spectrometers when using detectors with higher detection efficiency for low energy photons. This includes devices such as the n-type HPGe detector with a very thin dead layer.

Table 1 shows the range of the main gamma-ray energies to be produced by $\mathrm{X}-\gamma$ summing for the $\mathrm{n}$-type HPGe detector with the commercial calibration source. It should be nearly attenuated to $40 \mathrm{keV} \mathrm{X-ray} \mathrm{from}{ }^{139} \mathrm{Ce}$ to avoid the X- $\gamma$ summing effect during gamma-ray spectrometry. As shown in Figure 1B, the photon's total linear attenuation rate for about $40 \mathrm{keV}$ is nearly $99 \%$ in $1 \mathrm{~mm}$-thick $\mathrm{Cu}$ with the general density of $8.96 \mathrm{~g} \cdot \mathrm{cm}^{-3}$. To minimize the decrease in detection efficiency, the optimal thickness was determined to be $1 \mathrm{~mm}$. A Cu shield was made and attached to the bottom of a cylindrical sample holder for practical use, as shown in Figure 2.

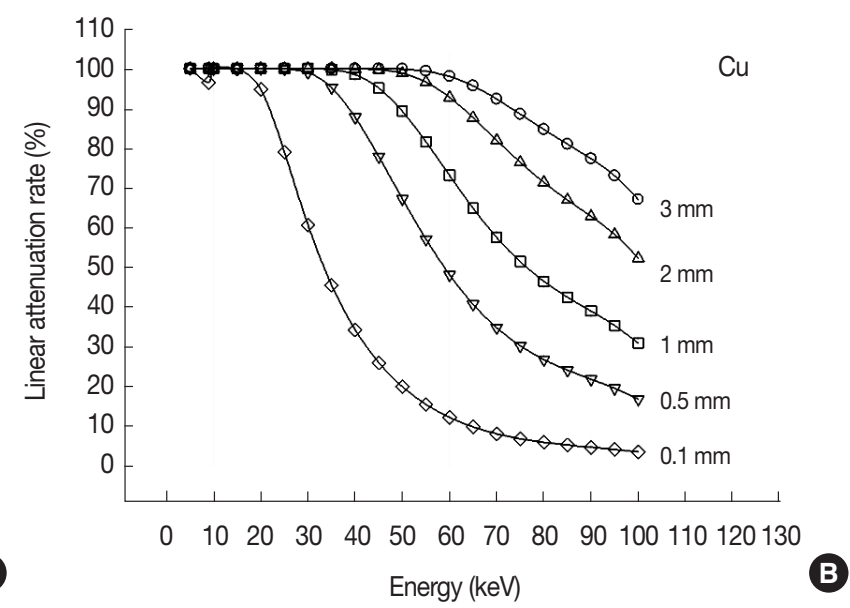

Fig. 1. (A) Ratio of photoelectric and total mass attenuation rates and (B) photon linear attenuation rate excluding coherent scattering in $\mathrm{Cu}$ $\left(\rho=8.96 \mathrm{~g} \cdot \mathrm{cm}^{-3}\right)$.

1) Berger MJ, Hubbell JH, Seltzer SM, Chang J, Coursey JS, Sukumar R, Zucker DS, Olsen K. XCOM: Photon Cross Section Database (version 1.3). 2005. 
Table 1. Main Gamma Ray Energy Peaks Produced by X- - Summing in the Standard Mixed Gamma Source

\begin{tabular}{|c|c|c|c|c|c|c|c|}
\hline \multirow[b]{2}{*}{ Nuclide } & \multirow{2}{*}{$\begin{array}{c}\text { X-ray } \\
\text { Energy* } \\
\text { (keV) }\end{array}$} & \multicolumn{3}{|c|}{ Gamma ray-1 } & \multicolumn{3}{|c|}{ Gamma ray-2 } \\
\hline & & $\begin{array}{c}\text { Energy } \\
(\mathrm{keV})\end{array}$ & $\begin{array}{c}\text { Intensity } \\
(\%)\end{array}$ & $\begin{array}{c}\text { Summed peak } \\
(\mathrm{keV})\end{array}$ & $\begin{array}{c}\text { Energy } \\
(\mathrm{keV})\end{array}$ & $\begin{array}{c}\text { Intensity } \\
(\%)\end{array}$ & $\begin{array}{c}\text { Summed peak } \\
(\mathrm{keV})\end{array}$ \\
\hline \multirow[t]{2}{*}{${ }^{57} \mathrm{Co}$} & 6.4 & 122.1 & 85.5 & 128.5 & 136.5 & 10.7 & 142.9 \\
\hline & 7.1 & 122.1 & 85.5 & 129.1 & 136.5 & 10.7 & 143.6 \\
\hline \multirow[t]{3}{*}{${ }^{139} \mathrm{Ce}$} & 33.2 & 165.9 & 79.9 & 199.1 & & & \\
\hline & 37.9 & 165.9 & 79.9 & 203.8 & & & \\
\hline & 38.8 & 165.9 & 79.9 & 204.7 & & & \\
\hline \multirow[t]{3}{*}{${ }^{85} \mathrm{Sr}$} & 13.4 & 514.0 & 98.5 & 527.4 & & & \\
\hline & 15.0 & 514.0 & 98.5 & 529.0 & & & \\
\hline & 15.2 & 514.0 & 98.5 & 529.2 & & & \\
\hline \multirow[t]{3}{*}{${ }^{88} Y$} & 14.1 & 898.0 & 93.7 & 912.1 & $1,836.1$ & 99.2 & $1,850.2$ \\
\hline & 15.9 & 898.0 & 93.7 & 913.9 & $1,836.1$ & 99.2 & $1,852.0$ \\
\hline & 16.1 & 898.0 & 93.7 & 914.1 & $1,836.1$ & 99.2 & $1,852.2$ \\
\hline
\end{tabular}

*Mean value.

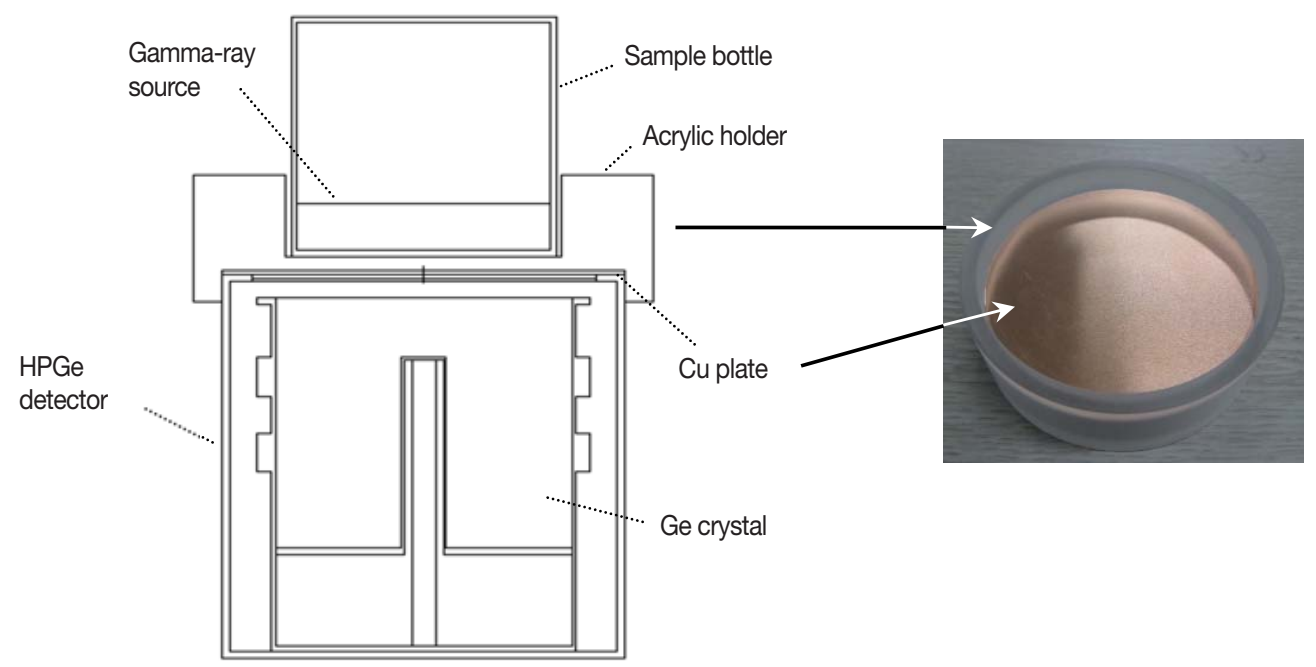

Fig. 2. Geometrical model of the n-type HPGe detector system with a $1 \mathrm{~mm}$-thick Cu shield using the MCNPX code.

The efficiency curves were compared for cylindrical standard sources $10 \mathrm{~mm}$ thick, with and without the $\mathrm{Cu}$ shield. The detection efficiency without $\mathrm{X}-\gamma$ summing effects was simulated to confirm the effectiveness of the $\mathrm{Cu}$ shield comparing with measured efficiency. To simulate the detection efficiency without $\gamma-\gamma$ summing effects, a Monte Carlo simulation using the MCNPX code was conducted; the geometrical model is shown in Figure 2 [3-5]. The pulse height tally 'F8:P' with default physics option in the code was used to simulate the detection efficiency for the detector. The simulation was validated by comparing the measured and simulated results for ${ }^{241} \mathrm{Am},{ }^{109} \mathrm{Cd},{ }^{203} \mathrm{Hg}$, and ${ }^{137} \mathrm{Cs}$ contained in the standard source because the true coincidence summing can be neglected for the nuclides with the detector and gamma source used in this study. The true coincidence summing correction factors with and without the $\mathrm{Cu}$ shield were also calculated for ${ }^{57} \mathrm{Co},{ }^{139} \mathrm{Ce},{ }^{85} \mathrm{Sr},{ }^{60} \mathrm{Co}$, and ${ }^{88} \mathrm{Y}$ in order to doublecheck the summing effect. The general formula for the factor (CF) can be expressed as

$$
\frac{1}{C F}=\frac{n^{\prime}}{n}=1-\sum_{i=1}^{N} f_{i} \varepsilon_{t i}
$$

where

$n$ : gamma-ray count rate without the true coincidence summing,

$n$ ': gamma-ray count rate with the true coincidence summing,

$N$ : total number of photons coincided ('within the resolving time') with gamma-ray of interest, 

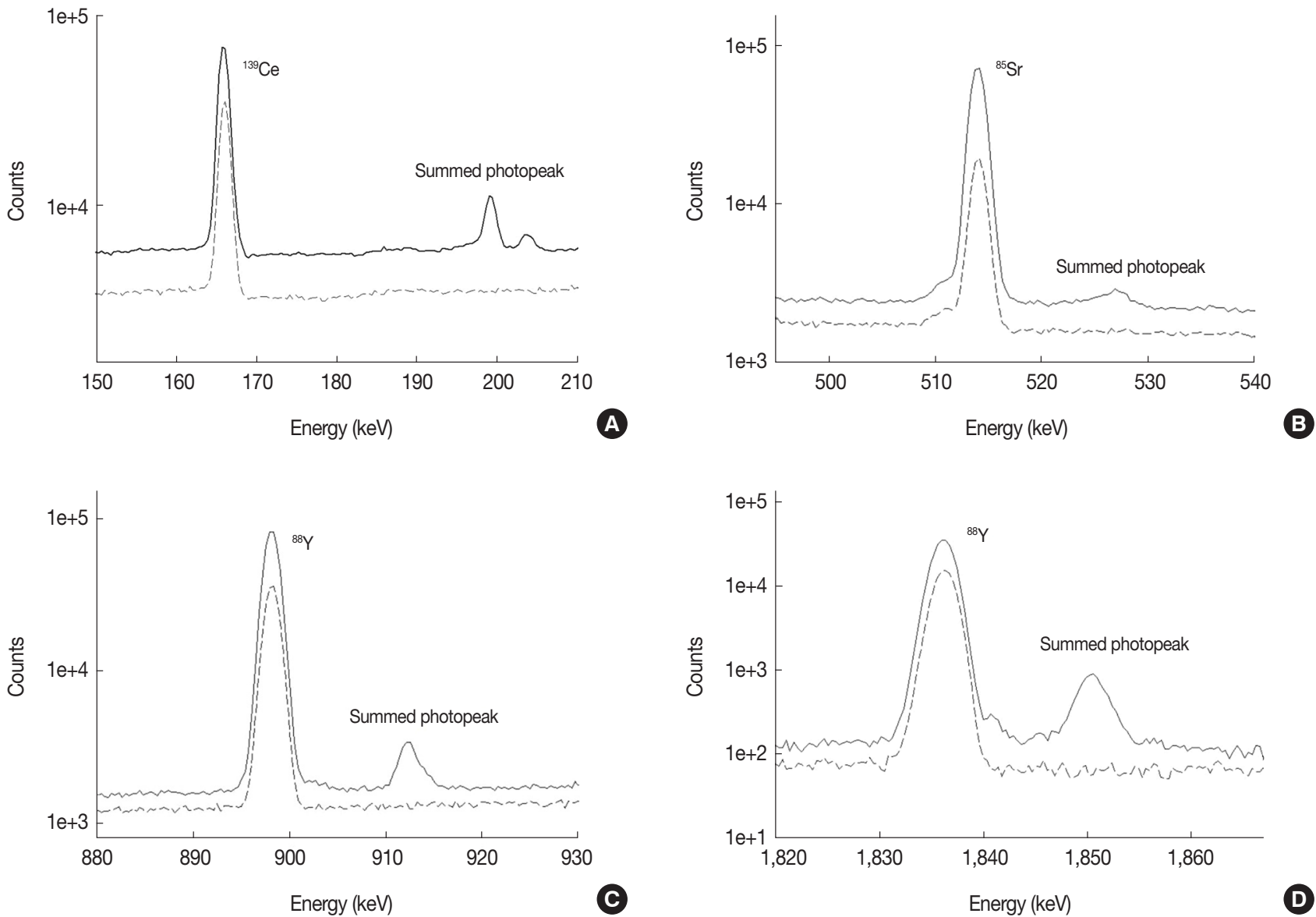

Fig. 3. Pulse-height spectra obtained for a calibration source with (---) and without (-) the $1 \mathrm{~mm}$-thick Cu shield.

$f_{i}$ emission fraction of $i$-th photon coincided with gammaray of interest,

$\varepsilon_{t i}:$ total efficiency for $i$-th photon coincided with gammaray of interest.

The internal conversion coefficients, electron capture probability and K-shell X-ray fluorescence needed for $f_{i}$ were referenced the nuclear data from the Recommended Data $(\mathrm{LNHB})^{2)}$, and $\varepsilon_{t i}$ was obtained by the Monte Caro simulation in the same way mentioned above.

\section{Results and Discussion}

Figure 3 shows gamma-ray energy spectra with and without the $1 \mathrm{~mm}$-thick $\mathrm{Cu}$ shield, and the $\mathrm{X}-\gamma$ summing peaks for ${ }^{139} \mathrm{Ce},{ }^{85} \mathrm{Sr}$ and ${ }^{88} \mathrm{Y}$ measured with the shield are not observed in the spectra because of X-ray filtering in contrast to ones without a shield. Figure 4 and Table 2 represent the mea-

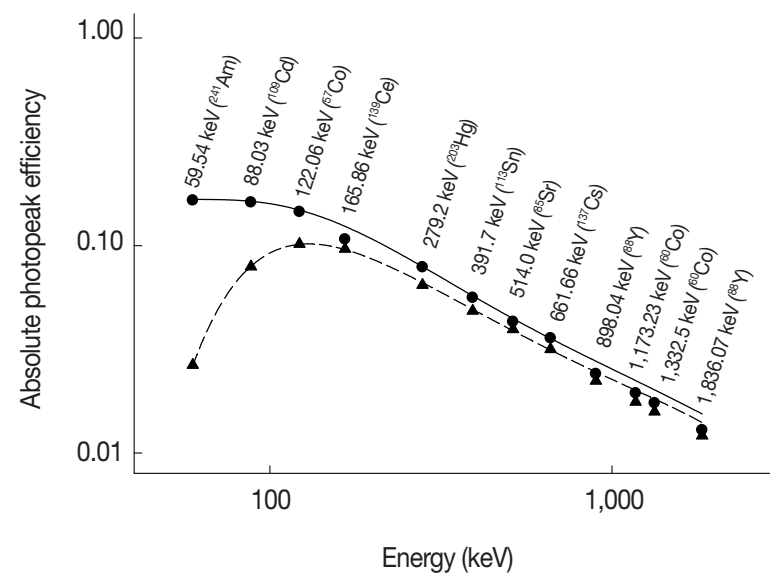

Fig. 4. Detection efficiencies measured and simulated for the n-type HPGe detector systems with and without the $1 \mathrm{~mm}$-thick Cu shield: (-) measured without a shield, (-) simulated and 6th-order fitted curve without a shield, ( $(\mathbf{\Delta})$ measured with the $1 \mathrm{~mm}$-thick Cu shield, (---) simulated and 6th-order fitted curve with the 1 mm-thick $\mathrm{Cu}$ shield.

\footnotetext{
2) Laboratory National Henri Becquerel, Recommended Data, http://www.nucleide.org/DDEP_WG/DDEPdata.htm.
} 
Table 2. Measured and Simulated Detection Efficiencies for the n-type HPGe Detector System with and without the $1 \mathrm{~mm}$-thick Cu Shield

\begin{tabular}{|c|c|c|c|c|c|c|c|c|c|}
\hline \multirow{2}{*}{ Nuclide } & \multirow{2}{*}{$\begin{array}{c}\text { Energy } \\
(\mathrm{keV})\end{array}$} & \multicolumn{4}{|c|}{ Efficiency without the Cu shield } & \multicolumn{4}{|c|}{ Efficiency with the Cu shield } \\
\hline & & Measured* $^{*}$ & Simulated & Simul./Meas. & $\mathrm{CF}^{\dagger}$ & Measured $^{*}$ & Simulated & Simul./Meas. & $\mathrm{CF}^{\dagger}$ \\
\hline${ }^{241} \mathrm{Am}$ & 59.5 & $1.65 \times 10^{-1}(0.76)$ & $1.67 \times 10^{-1}$ & 1.01 & - & $2.65 \times 10^{-2}(0.82)$ & $2.63 \times 10^{-2}$ & 0.99 & - \\
\hline${ }^{109} \mathrm{Cd}$ & 88.0 & $1.61 \times 10^{-1}(2.86)$ & $1.63 \times 10^{-1}$ & 1.01 & - & $7.89 \times 10^{-2}(2.87)$ & $7.96 \times 10^{-2}$ & 1.01 & - \\
\hline${ }^{57} \mathrm{Co}$ & 122.1 & $1.45 \times 10^{-1}(0.73)$ & $1.48 \times 10^{-1}$ & 1.02 & 1.01 & $1.02 \times 10^{-2}(0.95)$ & $1.01 \times 10^{-2}$ & 0.99 & 1.00 \\
\hline${ }^{139} \mathrm{Ce}$ & 165.9 & $1.07 \times 10^{-1}(0.75)$ & $1.24 \times 10^{-1}$ & 1.16 & 1.16 & $9.62 \times 10^{-2}(1.07)$ & $9.57 \times 10^{-2}$ & 0.99 & 1.00 \\
\hline${ }^{203} \mathrm{Hg}$ & 279.2 & $7.85 \times 10^{-2}(0.72)$ & $7.92 \times 10^{-2}$ & 1.01 & - & $6.43 \times 10^{-2}(2.69)$ & $6.62 \times 10^{-2}$ & 1.03 & - \\
\hline${ }^{113} \mathrm{Sn}$ & 391.7 & $5.59 \times 10^{-2}(2.01)$ & $5.69 \times 10^{-2}$ & 1.02 & - & $4.82 \times 10^{-2}(2.11)$ & $4.89 \times 10^{-2}$ & 1.01 & - \\
\hline${ }^{85} \mathrm{Sr}$ & 514.0 & $4.28 \times 10^{-2}(1.92)$ & $4.44 \times 10^{-2}$ & 1.04 & 1.02 & $3.93 \times 10^{-2}(1.33)$ & $3.86 \times 10^{-2}$ & 0.98 & 1.00 \\
\hline${ }^{137} \mathrm{Cs}$ & 661.7 & $3.56 \times 10^{-2}(0.82)$ & $3.56 \times 10^{-2}$ & 1.00 & - & $3.15 \times 10^{-2}(0.87)$ & $3.12 \times 10^{-2}$ & 0.99 & - \\
\hline${ }^{88} Y$ & 898.0 & $2.40 \times 10^{-2}(0.72)$ & $2.76 \times 10^{-2}$ & 1.15 & 1.16 & $2.22 \times 10^{-2}(0.88)$ & $2.46 \times 10^{-2}$ & 1.11 & 1.12 \\
\hline${ }^{60} \mathrm{Co}$ & $1,173.2$ & $1.93 \times 10^{-2}(0.77)$ & $2.23 \times 10^{-2}$ & 1.16 & 1.14 & $1.76 \times 10^{-2}(0.85)$ & $2.00 \times 10^{-2}$ & 1.14 & 1.13 \\
\hline${ }^{60} \mathrm{Co}$ & $1,332.5$ & $1.74 \times 10^{-2}(0.77)$ & $2.01 \times 10^{-2}$ & 1.16 & 1.14 & $1.58 \times 10^{-2}(0.85)$ & $1.81 \times 10^{-2}$ & 1.15 & 1.14 \\
\hline${ }^{88} Y$ & $1,836.1$ & $1.28 \times 10^{-2}(0.73)$ & $1.54 \times 10^{-2}$ & 1.20 & 1.19 & $1.21 \times 10^{-2}(0.93)$ & $1.40 \times 10^{-2}$ & 1.16 & 1.14 \\
\hline
\end{tabular}

${ }^{*}$ Relative uncertainties (\%) with $k=1$ are given in brackets in each column.

'True coincidence summing correction factor calculated in this study.

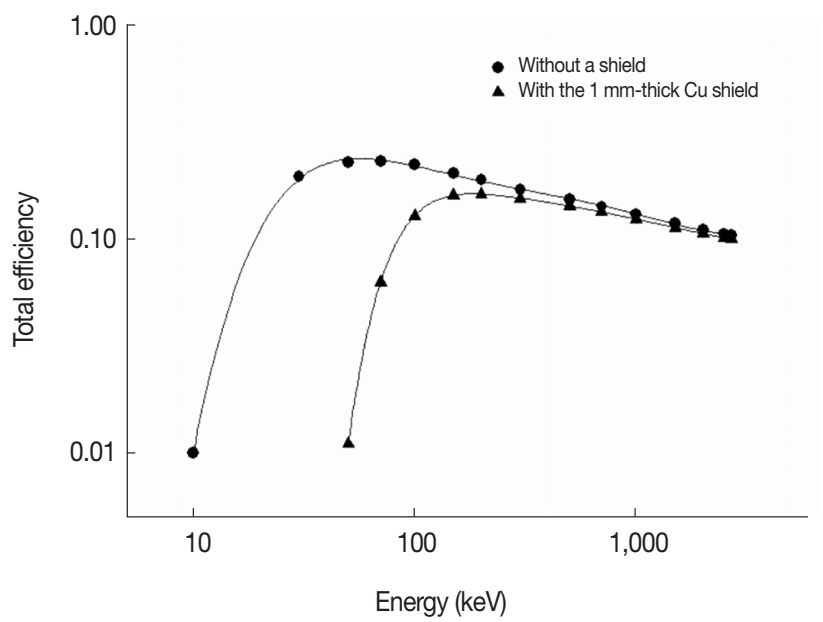

Fig. 5. Total efficiency of an n-type HPGe detector for a liquid volume source $\left(4.8 \varnothing \times 1 \mathrm{~cm}^{3}\right)$ with and without the $1 \mathrm{~mm}$-thick Cu shield.

sured and simulated detection efficiencies of the cylindrical source $\left(4.8 \emptyset \times 1 \mathrm{~cm}^{3}\right)$ with and without, respectively, true coincidence summing. The absolute efficiencies for the fullenergy peaks by measurement and simulation with and without the $\mathrm{Cu}$ shield for ${ }^{241} \mathrm{Am},{ }^{109} \mathrm{Cd},{ }^{203} \mathrm{Hg}$, and ${ }^{137} \mathrm{Cs}$ agreed well (within 3\%). Figure 5 shows the simulated total efficiency, and there was good agreement between the calculated correction factor (CF) and the ratio of measured and simulated efficiency within $3 \%$. This shows that the validity of the simulation was demonstrated. For the measurement system without a $\mathrm{Cu}$ shield, differences between the measured and simulated efficiencies for ${ }^{139} \mathrm{Ce},{ }^{85} \mathrm{Sr}$, and ${ }^{88} \mathrm{Y}$ were larger than for other nuclides because of $X-\gamma$ summing. The greatest difference was for ${ }^{88} \mathrm{Y}$ due to $\mathrm{X}-\gamma$ summing and $\gamma-\gamma$ summing. In contrast, ${ }^{241} \mathrm{Am},{ }^{109} \mathrm{Cd},{ }^{57} \mathrm{Co},{ }^{203} \mathrm{Hg},{ }^{113} \mathrm{Sn}$, and ${ }^{137} \mathrm{Cs}$ had small differences compared to ${ }^{139} \mathrm{Ce}$, ${ }^{85} \mathrm{Sr}$, and ${ }^{88} \mathrm{Y}$ because the true coincidence summing for the nuclides can be neglected in the spectrometer used in this study. The $\mathrm{X}-\gamma$ summing for ${ }^{57} \mathrm{Co}$ with the present volume source can be neglected, but it can be occurred significantly for a point source or thin paper filter source close to the detector which has high detection efficiency for low energy. The measured efficiencies with the $\mathrm{Cu}$ shield agreed with the simulated ones within $3 \%$ except for ${ }^{88} Y$ and ${ }^{60} \mathrm{Co}$. Due to $\gamma-\gamma$ summing, ${ }^{88} \mathrm{Y}$ and ${ }^{60} \mathrm{Co}$ had larger differences than the other nuclides. The results show that the $\mathrm{X}-\gamma$ summing effect can be avoided using a $\mathrm{Cu}$ shield of appropriate thickness, and that the accuracy of the detection efficiency calibration for gamma-ray detectors with windows of thin or low atomic materials can be improved.

\section{Conclusion}

In this study, the thickness of a $\mathrm{Cu}$ shield needed to avoid true coincidence summing effects due to $X-\gamma$ was tested and determined to be $1 \mathrm{~mm}$, considering the detection efficiency desired for higher energy. As a result, the accuracy of the detection efficiency calibration was improved by more than $13 \%$ by reducing $\mathrm{X}-\gamma$ summing. Although gamma-ray detectors with a window consisting of thin or low atomic number materials (such as an n-type HPGe detector) are used for lower energy gamma-ray measurements, the use of appropriate shields can improve the applicability and accuracy of such detectors for high energy gamma-rays. The X- $\gamma$ summing effect should be considered, along with $\gamma-\gamma$ summing, 
when a detection efficiency calibration is implemented. This is particularly true for gamma-ray detectors having photon incidence windows with thick or low atomic number materials. In such cases, appropriate shielding material such as $\mathrm{Cu}$ can be useful for simplifying analysis of the gamma-ray energy spectrum during detection-efficiency calibration and for improving the quantification of radioactivity in test samples by avoiding $X-\gamma$ summing effects.

\section{References}

1. Debertin K, Helmer RG. Gamma- and X-ray spectrometry with semiconductor detectors. 3rd Ed. Amsterdam, Netherlands. NorthHolland. 1988;258-270.
2. Montgomery DM, Montgomery GA. A method for assessing and correcting coincidence summing effects for germanium detector efficiency calibrations. J. Radioanal. Nucl. Chem. 1995;193(5): 71-79.

3. Sima O, Arnold D. Self-attenuation and coincidence-summing corrections calculated by Monte Carlo simulations for gammaspectrometric measurement with well-type germanium detectors. Appl. Radiat. Isot. 1996;47(9-10):889-893.

4. García-Toraño E, Pozuelo M, Salvat F. Monte Carlo calculations of coincidence-sum corrections for volume sources in gammaray spectrometry with Ge detectors. Nucl. Instrum. Methods Phys. Res. Sect. A. 2005;554(3):577-583.

5. Venkataraman R, Croft S, Russ WR. Calculation of peak-to-total ratios for high purity germanium detectors using Monte-Carlo modeling. J. Radioanal. Nucl. Chem. 2005;264(4):183-191. 